\title{
A Topology Control Method with Prediction of Lossy Links in Cluster Based Mobile Ad-Hoc Networks
}

\author{
T. Parameswaran ${ }^{1}$, C. Palanisamy ${ }^{2}$, Anand Paul $^{3,}{ }^{*}$ and Seungmin Rho ${ }^{4}$ \\ ${ }^{1}$ Department of CSE, Anna University Regional Centre,, India \\ ${ }^{2}$ Department of IT, Bannari Amman Institute Of Technology, India \\ $3,{ }^{*}$ School Of Computer Science and Engineering, Kyungpook National \\ University, Korea \\ ${ }^{4}$ Department Of Multimedia, Sungkyul University, Anyang-Si, Korea \\ ${ }^{1}$ Tparameshse@Gmail.Com, ${ }^{2}$ Cp_Samy@Yahoo.Com, \\ 3,*Paul.Editor@Gmail.Com, ${ }^{4}$ smrho@Sungkyul.Ac.Kr
}

\begin{abstract}
Many effects have been applied to maintain the stable topology and increase the stability of the Mobile Ad-Hoc Networks (MANET). Traditional Topology control mechanisms fail to consider lossy links which provide only probabilistic connectivity. The existing methods such as cooperative communication, Distributed Topology Control method, and Joint authentication control method have not ensured the survivability of a node. The high possibility of a link breakdown due to low energy in ad-hoc networks causes the cluster head selection issues with regard to achieving the best possible service reliability. We propose Cluster Dependent Topology Control (CDTC) Framework, which provides the ability to elect a cluster head by taking few metrics such as the residual energy of the cluster head, the lifetime of a cluster head within a cluster and network reachability. We attempt to find the minimal survivability of nodes under the conditions of the dynamic movement of individual nodes which will ensure that a set of mobile nodes form a connected network. Our proposed method is on-demand, which balances link failure, improves the network connectivity, and prolongs the lifetime of MANETs.
\end{abstract}

Keywords: Topology, Cluster Head, Stability, Survivability, Reliability

\section{Introduction}

The MANET is usually formed by a set of mobile nodes instantly, which can communicate among themselves by means of air medium. These nodes are creating a network dynamically without relying on the support of any infrastructure and cooperate by forwarding the data between the nodes among themselves through of single or multi-hop links without any central administration. This flexibility enables them to be used whenever the fixed infrastructure is unfeasible. Hence the MANET is very useful in the fields such as military battle field communication, sensing, or controlling a region, disaster relief, sharing information during the conference or seminar etc. The node-to-node links are established and broken quite often because of various reasons including dynamic topology, node failure, mobility, and deliberate changes in the transmission power of the nodes.

The way to reduce the power consumption of each node can be achieved by means of adjusting the transmission range which potentially reduces the energy consumption involved during the transmission. Moreover, reducing the transmission power will eliminate the long distance links that can avoid the waste of resources, reduction in packet retransmission and interference. Additionally, the network 
overhead, scalability, interference with neighboring node or adjacent cluster and handling of vulnerable nodes in large network will affect the performance of the network. Hence the maintenance of fully connected topology in the networks needs much attention in certain situations.

A topology gives information of connected nodes and their connectivity (link) status between the pair of connected nodes. Here each node has information about its neighbor and make a relative virtual link to construct a network. The idea of topology control is to grant the mobile nodes a sense of control over certain parameters such that these parameters can be manipulated in a way that improves the stability of a network.

Many of the authors $[7,10,13,22]$ interchangeably consider the topology control as a technique which adopts the power saving scheme by adjusting the transmission range of nodes and clearly excludes clustering approaches. Here we are proposing a clustering method to maintain a sustained topology with a minimum duration in a dynamic environment. In clustering dependent topology control approach, mobile nodes select a set of nodes to form a cluster. This method provides a control over topology to achieve a persistent topology by means of 1) Estimating the cluster head life time 2) Estimating Individual nodes life time within the cluster called Survivability 3) Admission control over the cluster in case the entry of new node 4) Prediction and control of node movements by measuring the relative speed with respect to cluster head 5) Estimation of lossy links within the cluster. Under the assumption of success of the above factors, the overall method is shown to improved connectivity of the mobile network.

The remaining of the work is organized as follows. In section II, we discuss about related work associated with the proposed problem. In section III, we define the problem formulation for creating and electing the cluster and Cluster Head $(\mathrm{CH})$. In section IV, we focus on estimating the lossy links within the cluster to avoid the retransmission of packets. Finally, in Section V, we illustrate the result of this combined framework and present the improvement of topology stability. The consideration of vulnerability in a cluster is beyond the scope of this work.

\section{Related Works}

\subsection{Clustering}

Creating of virtual group of nodes with a volatile time period in MANET is called Clustering. Here the nodes are allocated geographically adjacent to each other in the same cluster by adhering to certain rules with depending on the application. The probabilistic network model is proposed by Y. Liu et al [1]. In data centric approach this model ensures only the network reachability, transmission range, and energy. The system does not consider about link stability and its qualities. Moreover, the probabilistic based models give the approximated results in network stability and topology control. G. Guan et al [2] jointly consider topology control and node authentication method. In the event time simulation, the Joint Authentication Topology Control (JATC) provides improved throughput by the formulation of discrete stochastic optimization approaches. Here the communication reliability and efficiency has been improved by considering transmission power, channel assignment, antenna direction, and type of transmission parameters are used to maintain the link status will be active in the request time.

In spite of the all centralized methods few of the localized methods have focused on the Directed Local Spanning Subgraph [8, 12] and Directed Relative Neighborhood Graph were implemented for heterogeneous wireless network. In which the dynamic mobility creates a challenge for constructing the spanning 
subgraph. Moreover, these systems have lack of control over the measure of lossy link to avoid packet loss.

Li describes cone based topology control algorithm, which focuses on finding the nodes in the transmission direction, instead of finding a node location. This algorithm provides some useful properties like symmetry, sparseness, and good routes. But this system fails to address the problems in a dynamic environment. On the dynamic environment both centralized and distributed approaches focus on maintaining strong connectivity at the expense of scalability. To resolve this problem, C.C. Shen et al [4] have proposed the cluster-based topology control algorithm. This work is mainly focused on adjusting the transmission power to control the link failure problem.

However, the adjustment of the transmission power may increase interference among the neighboring node within a cluster and among adjacent cluster groups. Moreover, this system does not consider the about dynamic movement of nodes with varying speed.

\subsection{Admission Control and Survivability}

The lack of centralized control, cooperation between nodes, unreliable wireless channel, and node mobility are combined together and introduce new challenges to QoS parameters in MANET [6]. The admission control schemes have provided an optimized solution with certain limitations by means of highlighting the characteristics such as channel capacity, buffer size, battery charge, and processor time. Moreover, these parameters give the records to stateful/stateless protocols.

The survivability of a network or node is defined as its fulfilling the mission, in a timely manner in the presence of link failure, several attacks, or accidents [5]. In the area of defense, firewall, cryptography techniques, access control, and Virtual Private Network (VPN) are very helpful to increase the network survivability.

\subsection{Lossy Link Estimation}

The use of geographic routing provides a mechanism to control over the monitoring of the link between nodes in wireless sensor networks [9]. The a-priori or self-reconfiguring mechanism provides location information of the node and its links. Here the blacklisting / neighbor selection scheme is used to avoid weak links in the network. Distance-hop Energy Trade-off is used to increase the range of communication for large hop transmission. The whole approach has been focused on sensor networks. But this system fails to address the issues of mobile sensor nodes.

Reconfiguring of topology based on traffic engineering scheme, generates the statistical report on traffic monitoring, bandwidth prediction, and reconfiguration trigger, topology design \& topology migration. The connectivity based topology control method operates in either connected or disconnected state. The network in which all the nodes are connected is connected as fully connected [11, 14, 15, 21]. In the reconfiguration scheme, the connectivity based topology control gives linear network reachability rather than opportunity based topology control which provides exponential network reachability. In other words, the components of the network such as, links and nodes that are fully correlated to each other are called as a coherent system. Some of the reconfiguration methods are based on energy level between the connected pair of nodes. From the research paper considered so far, it can be concluded that the network reachability should be a fully distributed manner over the network. 


\section{Topology Control}

\subsection{System Model for Topology Control}

The network topology control is used to determine the deployment of the node links and to make an effective link between the nodes to form a good topology. Many of the distributed topology control methods do not effectively help in the collection of node information in the network. To overcome the above issue, we proposed distributed topology is being modeled by means of clustering.

Let us consider a network that has $\mathrm{N}$ number of clusters and all the cluster members are fully connected with the $\mathrm{CHs}$ in each cluster and can be modeled as

$$
C g_{n}^{*}=N \text { arg max } f\left(C g_{n}\right)
$$

Where

$$
\begin{aligned}
& C g_{n}=G(\vartheta, \varepsilon) \\
& \vartheta \text { is the number of nodes in the cluster, and } \\
& \varepsilon \text { is the number of link connections between the nodes, } \\
& N \text { denotes number of clusters in the network }
\end{aligned}
$$

Generally, the topology control can be achieved by adjusting network parameters such as transmission power, antenna parameters like gain and beam width, antenna direction, channel assignment, node cooperation level etc. Apart from adjusting these parameters, in case of random movements of nodes, the cooperative methods fail to adhere to the topology control properties.

To avoid these problems, we have given importance to the following factors while designing a mobile network.

- All nodes must belong to at least one cluster.

- Node degrees of the cluster head should be kept at a minimum for lossy less transmission.

- The hop count of the network should be reduced.

- The transmission radius should be reduced to avoid interference between clusters.

- Guarantee connectivity to avoid lossy links and multiple retransmissions.

- Minimize the number of bi-connected components to reduce the excessive bandwidth utilization.

- Maximize the size of the largest bi-connected component to establish coverage of the whole cluster.

- Maximize the distance between the cluster heads for frequency spectrum reuse.

\section{A. Topology Construction}

The objective of the proposed method is to create a topology with clusters and nodes that should be aware of its mobility and its lifetime. Towards this end, the Cluster Dependent Topology Control (CDTC) method includes i) Calculation of Mobility, ii) Calculation of nodes Relative Speed, iii) Estimation of nodes Life Time, iv) calculation of residual energy. Many of the research results $[2,3,4,8]$ conclude that the stable topology environment can be achieved by means of adjusting the transmission parameters. In case of dynamic environment, these results fail to ensure the minimum survivability of the nodes within the cluster.

\section{B. Mobility Calculation}

The CDTC framework maximizes the cluster stability by choosing the $\mathrm{CH}$ with relatively low mobility. The movements of each node are unrestricted. i.e., the 
trajectories of the node can be anywhere in the topographical area. We represent node movement in two dimensional representation, i.e., $\vartheta=(x, y)$.

The mobility of a node can be calculated by the average of the distances covered in its last $n$ time slots. The total distance $d_{t}$ travelled by a node in time $t$ is

$$
d_{t}=\sum_{i=t-n}^{i=t} \text { dist }_{i}
$$

Where,

$\mathrm{i}=\mathrm{t} \rightarrow$ current time

The node mobility can be calculated using the relation

$$
N_{m o b}=\frac{d_{t}}{n}
$$

\section{Estimation of Residual Energy}

The residual energy is remaining energy that a node does not able to send or receive data Energy level of each node should be above the threshold value of the residual energy. The node cannot be admitted to the cluster if its energy level becomes lower than residual energy. The energy level of an idle node can be calculated as

$$
E_{\text {idle }}=M * \text { Size }_{p k t}+C_{\text {bcast }}
$$

Where,

$\mathrm{M}$ is incremental cost to send or receive a packet

$\mathrm{C}$ fixed cost for broadcast communication

It is differs for cluster head with some basic assumptions.

- Number of Cluster Members(CM) associated with $\mathrm{CH}$ during communication

- Cumulative transmission power utilized by $\mathrm{CH}$ to provide service to the $\mathrm{CH}$ members.

- Total traffic associated with the $\mathrm{CH}$.

Total Energy consumed by $\mathrm{CH}$ can be calculated as

$$
E_{\text {ch }}=\alpha * \| n_{i} \mid+\beta * T F_{b c a s t}+\gamma * \sum_{v \in i} \operatorname{dist}(u, v)
$$

Where $\left|n_{\mathrm{i}}\right|$ - Cardinality of the cluster

$\mathrm{TF}_{\text {bcast }}$ - cost of energy consumption in traffic forwarding

$\sum_{\mathrm{v} \in \mathrm{i}} \operatorname{dist}(\mathrm{u}, \mathrm{v})-$ Total transmission power.

$\alpha, \beta \& \gamma$ are weights assigned based on the network density.

If network traffic increases then the weight of the $\beta$ will be high. In case of dense network cardinality value will be high and also the value of $\alpha$ will be higher than that of remaining weights.

\section{Cluster Formation}

In infrastructure less network, the nodes control and cooperation have predefined role in the network. But it is not achieved in real time network. The cluster creation gives more specific approach to have a control over mobile nodes in many mobility models.

The single hop cluster gives more reliable communication among the nodes and offer value added services in the network. To form a cluster in MANET,

- Number of nodes in the network. 
- Node degree with single hop connection.

- Number of nodes in the cluster will lies between upper and lower limit values.

- Admission control over the joining of new node.

\section{E. Cluster Size Prediction}

In cluster based network environment, the cluster size to be predefined. This will step into reducing the lack of communication between the $\mathrm{CH}$ and $\mathrm{CM}$. Moreover, the packet retransmission, packet loss, problems with lossy links also be reduced by fixing the cluster size value. The criteria for cluster size are:

- Channel bandwidth: To be initialized during the period of network setup.

- Number of communication channel allocated to a respective cluster: in case of Frequency Division Multiplexing scheme (FDM), the number of communication channel and its guard band range is to be allocated.

- Maximum load handling capacity of a $\mathrm{CH}$ : determines the maximum number of single hop nodes connected to the $\mathrm{CH}$ to offer the service.

- Time limit of Idle/Busy status of the channel: to reduce the unwanted resources utilization of a channel and waiting time of service request node.

- Number of standby channels in the cluster: Minimum no of standby channels will determine the upper value of the cluster size.

- Minimum number of neighboring nodes around $\mathrm{CH}$ : This criterion will determine the lower degree of the cluster size.

\section{F. Cluster Head Election}

Cluster Head election process is needed for cluster based network. Many of the research work have been done on selection of cluster head in clustered network such as MANET, VANET, and WSN. The cluster head selection algorithms follows on the deterministic/ non deterministic clustering, on-demand based clustering, Lowest ID based clustering, and Connectivity based clustering, adaptive clustering. There are some limitations in the parameter utilization on cluster head selection method. To overcome these issues, the following steps will be followed for $\mathrm{CH}$ selection Process.

Step 1: Number of node count in the cluster

Step 2: Node status identification

Step 3: Estimation of Node life time within the cluster

Step 4: Energy level calculation

Step 5: Nodes Speed prediction

Step 6: Enabling the Election Process

Step 7: Verifying the $\mathrm{CH}$ nodes status

\subsection{Mobility Model}

In a MANET, the movements of the nodes are totally stateless. The future movement of a node is completely independent of the past movement. So the motion is purely unpredictable. The Gauss Markov model [20] creates the random movements with new speed and direction based on current speed and direction. This model updates the next movement of the mobile nodes. The proposed system considers the movements of the nodes with respect to cluster head. Many of the problems arise due to the sharp changes in the speed and direction. To avoid this problem, the speed and direction at time $t$ should be a function of the speed and direction at time $\mathrm{t}_{\mathrm{n}-1}$. Such that: 


$$
S_{n}=f\left(S_{n-1}\right) \& D_{n}=f\left(D_{n-1}\right)
$$

Where,

$\mathrm{S}_{\mathrm{n}}$ is speed of a node $\mathrm{n}$ and

$D_{n}$ is direction of a node $n$

Based on the Gaussian distribution, the speed and direction of a node are calculated at each interval by using the above formula. The new values of the direction and speed can be calculated at fixed interval of time by using the following equation:

$$
\begin{aligned}
& S_{n}=\alpha S_{n-1}+(1-\alpha) S^{\prime}+\sqrt{(1-\alpha)^{2}} * S_{x_{n-1}} \\
& D_{n}=\alpha D_{n-1}(1-\alpha) D^{\prime}+\sqrt{(1-\alpha)^{2}} * D_{x_{n-1}}
\end{aligned}
$$

Where,

$\alpha$ - to change the degree of the randomness of the model. If $\alpha \cong 0$, then randomness will increase and if $\alpha \cong 1$, then node movements will be sharp and model behaves like a linear movement model $\mathrm{S}_{\mathrm{x}_{\mathrm{n}-1}} \& \mathrm{D}_{\mathrm{x}_{\mathrm{n}-1}}$ are the random variables chosen from a Gaussian distribution. $\mathrm{S}^{\prime} \& \mathrm{D}^{\prime}$ are the mean speed and direction of the node movement. At each time the next coordinate of the mobile node is calculated by

$$
\begin{array}{r}
X_{n}=X_{n-1}+S_{n-1} \cos D_{n-1} \\
Y_{n}=Y_{n-1}+S_{n-1} \sin D_{n-1}
\end{array}
$$

Where, $X_{n} \& Y_{n}$ are the new coordinate of the mobile node.

\subsection{Node Current Table and Historical Table}

\section{Current Table}

Node_Table $\leftarrow$ Update $\{\#$ of Neighbors, Energy_value, linear/angular_velocity, TTL_Value $\left.{ }_{\mathrm{wC}}\right\}$

Historical table on each node

$\mathrm{Ht} \leftarrow$ \{Node_Id, Time_Update, Energy_Level, Avg_Speed, Relative Speed, Time_to_Expire WC $_{\text {, }}$ Node_Status,

Voting_Count, How_many_times_elected_as_CH_previously\}

Assigning Cluster Head Selection Priority

If node (Nodei $=$ Low_ mobility, TTL, High_voting_count, High_CH_count, Low_relative_speed, High_energy_value, Current_voting_count, High_residual_energy) \{

Priority = High; 
$\mathrm{CH}=$ Node $\left._{\mathrm{i}}\right\}$

\section{Topology Control Algorithm}

Input: No. of nodes in the network with Gauss Markov Mobility Model.

Output: No. of nodes with well-defined cluster and sustained topology control.

\section{Assumptions:}

- Nodes are active participants in the network

- $\quad$ No node is vulnerable

- All nodes are authorized.

- Initially, energy is randomly assigned between 10 to 100 joules.

Begin

- Each node must have its own historical data.

\{

//Create a network

$\mathbf{f}(\mathrm{G}(\theta, \varepsilon))$, where,

$\theta>2 \varepsilon>10$, otherwise

$/ / \theta=50$

// Channel status and broadcast message

If $($ Chstatus $=$ idle $)$

\{

Frame values $=\mathrm{F}\left(\mathrm{N}_{\mathrm{id}} ; \mathrm{N}_{\mathrm{sts}} \mathrm{I}=0 \| \mathrm{I}=1\right)$

Energy $=E(10 \leq E \leq 100)$

$\mathrm{B}_{\text {cast }}=$ Hello_Frame

Initiate=Reply_Count

Countn= $\mathrm{t} \geq 1$ TAckt

\}

elseif $\left(\mathrm{Ch}_{\text {status }}=\right.$ busy $)$

\{

Time $_{\text {wait }}++\leq \mathrm{WT}_{\text {th }}$

Check status;

\}

// Cluster Formation

\{

$\mathrm{N}_{\text {count }}=$ number of nodes in the network

if ( single hop $\mathrm{CM} \geq 2 \|$ Multi-hop $\mathrm{CM} \geq 2$ )

\{

Count $_{\text {neighbor_set }} \in \mathrm{Cg}$

$\mathrm{Cg}_{\text {Count }}=$ Count ++

\}

New_Node $\leftarrow\left\{\mathrm{N}_{\text {id }} ; \mathrm{N}_{\text {status }} ;\right.$ Energy $\left.; \mathrm{N}_{\text {speed }} ; \mathrm{N}_{\text {direction }}\right\}$

if $\left(\right.$ Count $\left._{\mathrm{CM}} \leq \mathrm{Th}\right)$

$\mathrm{CM} €$ New_Node

//Cluster Size Prediction

\{

$$
\begin{aligned}
& \mathrm{Ch}_{\mathrm{Bw}}=5 \mathrm{Mhz} \\
& \mathrm{Ch}_{\text {Comm }}=\text { number of } \mathrm{CM} \\
& \mathrm{CH}_{\text {Load }_{\max }}=\text { Count }_{\text {Singl Hop }}
\end{aligned}
$$




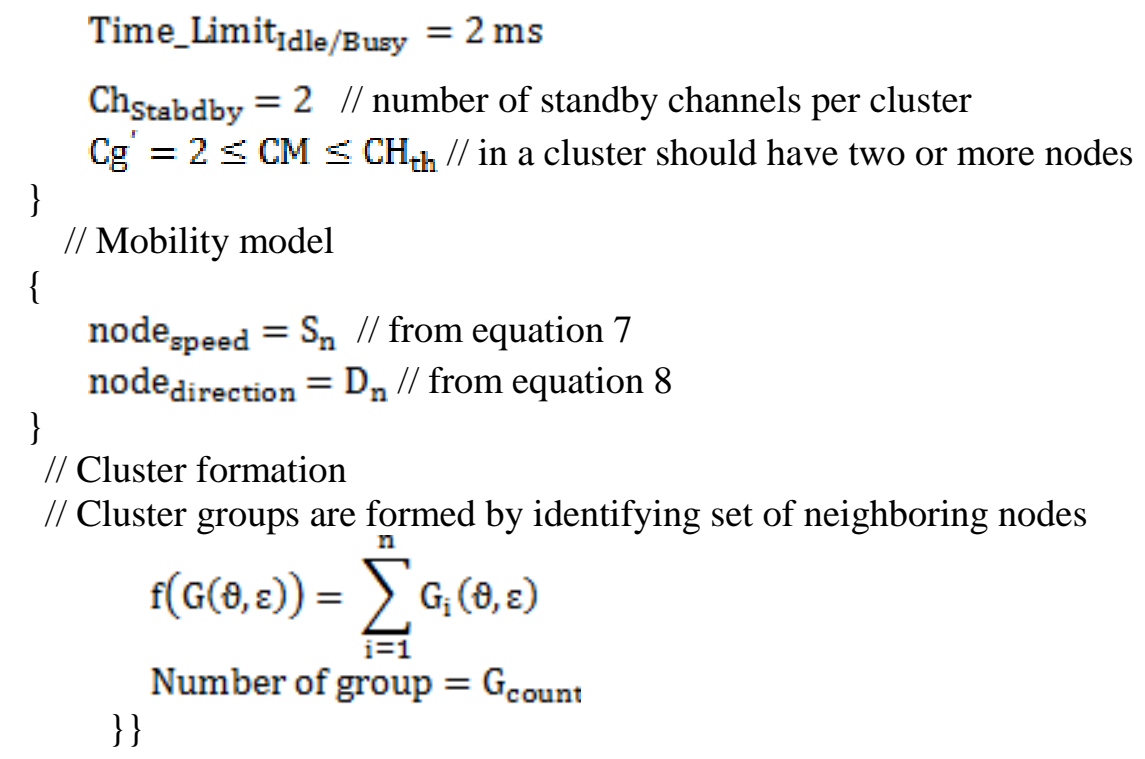

\subsection{Lossy Link Estimation}

Given the topology of unicast network and a packet loss threshold, we want to identify all links in the unicast network whose packet loss rates are greater than the threshold. We focus on identifying the lossy links using measurement mode with access to the source and receiver. A table is maintained to monitor all links in a network wherein we calculate the transmission rate and packet loss rate for all links. If any link results in high loss rate that is greater than the threshold value, then that link will be declared as a lossy link. This will be informed to the cluster head and alternate path will be identified to transmit the data.

\section{A. Identification of Lossy Link in a Cluster}

- Form a network based on cluster head with $\mathrm{V}$ as set of vertices or nodes and L as Link.

- Assign a threshold value $\alpha_{t h}, \epsilon$

- Initialize lossy link set as $L$ '.

- Calculate each node sequence according to

$$
X_{k}^{(i)}=V_{l \in R(k)} X_{k}^{(i)}
$$

If a node does not participate in transmission for long period, compare number of ' 0 ' sequence with that of its nearest node or path. Otherwise compare Hamming Distance of the node with other node, if $H_{d}(s 1, s 2)>\epsilon . n_{s}$, the link connecting number of ' 0 ' is more than other link then one of the link is considered as Lossy Link and added to L'.

\section{B. Hamming Distance Calculation}

Assumptions:

- Let $\mathrm{s}$ is the cluster head for a set of nodes $\left\{\mathrm{s}_{1}, \mathrm{~s}_{2}, \ldots \ldots \mathrm{s}_{\mathrm{n}}\right\}$ nodes.

- Let $\mathrm{n}_{\mathrm{s}}$ be the number of probe packets of the sender that are sent through cluster head s.

We use the following formula with XOR operator to find the distance between nodes of different paths 


$$
H_{d}\left(s_{1}, s_{2}, \ldots s_{n}\right)=\sum X_{s_{1}}^{1} \oplus X_{s_{2}}^{1} \oplus \ldots \oplus X_{s_{n}}^{1}
$$

Where, $\mathrm{H}_{\mathrm{d}}$ - Hamming Distance

\subsection{Network Stability Estimation}

The stability of the network is calculated through various metrics such as node velocity, received signal strength, and remaining node energy. In our proposed algorithm, we have used only node velocity and received signal strength parameters. The node velocity can be derived from the equations 7 and 8 and the network stability with respect to signal strength can be calculated through the following equation:

$$
S=\sum_{i=1}^{N} \frac{T_{n_{i-}} T_{f_{i}}}{S_{n_{i-}} S_{f_{i}}}
$$

Where, $\mathrm{N}$ is Number of Nodes. $\mathrm{S}_{\mathrm{n}_{\mathrm{i}}}$ Signal Strength if last received signal. $\mathrm{S}_{\mathrm{f}_{\mathrm{i}}}$

Signal Strength if first received signal. $\mathrm{T}_{\mathrm{n}_{\mathrm{i}}}$ Time taken for last signal reception. $\mathrm{T}_{\mathrm{f}_{\mathrm{i}}}$ Time taken for first signal reception.

\section{Simulation Results}

The proposed framework is simulated using NS2 simulator. The simulation environment presents $10 \%$ of random lossy links in the network. The simulation is carried out within $1000 \times 1000$ topological areas with three ranges of (20-100) node construction.

Table 1. Initial Parameters

\begin{tabular}{|l|l|}
\hline Parameter & Value \\
\hline Simulator & NS2.34 \\
\hline Channel & Wireless Channel \\
\hline Propagation & Shadowing \\
\hline Mac & 802.11 \\
\hline Queue & Drop Tail / Priority \\
\hline Antenna & Omni Antenna \\
\hline Topography & $1000 \times 1000$ \\
\hline Queue Length & 50 \\
\hline Mobility Model & Gaussian Markov \\
\hline Node Range & $20-100$ \\
\hline Simulation Time & 2000 \\
\hline
\end{tabular}

In our proposed work, we have evaluated with four phases of topology control framework. In the first one, we have validated the nature of the MANET behavior with regular topology. The second phase attempts the problems of cluster formation and cluster head selection schemes. After the selection of cluster head, the network realizes the un-stability of topology, which causes the problem of in-secure 
communication within the cluster. In the next phase we address this problem by including a monitoring scheme, which increases the stability of topology by adopting admission control over the nodes. The fourth phase estimates the link failure within the cluster in order to reduce the multiple re-transmissions of packets and thereby reducing the packet loss during communication. This scheme verifies the status of the link during the transmission instead of verifying at the beginning of the session.

Table 2. Current Table

\begin{tabular}{|c|c|c|c|c|c|}
\hline $\begin{array}{c}\text { Node } \\
\text { No }\end{array}$ & Time & $\begin{array}{c}\text { \# of } \\
\text { Neighbors }\end{array}$ & $\begin{array}{c}\text { Energy } \\
\text { level }\end{array}$ & $\begin{array}{c}\text { Avg. } \\
\text { Velocity (m/s) }\end{array}$ & $\begin{array}{c}\text { TTL within } \\
\text { cluster }\end{array}$ \\
\hline 5 & 0.2352 & 4 & 15.02 & 2.32 & 0.5342 \\
\hline 15 & 0.2568 & 5 & 10.45 & 1.38 & 0.7852 \\
\hline 20 & 0.3562 & 7 & 23.65 & 0.00 & 2.0000 \\
\hline 28 & 0.5362 & 3 & 41.23 & 2.92 & 0.3256 \\
\hline 35 & 0.7428 & 10 & 35.78 & 2.03 & 0.6785 \\
\hline 42 & 0.9258 & 6 & 26.13 & 1.92 & 0.6152 \\
\hline 48 & 1.1256 & 4 & 22.13 & 3.00 & 0.1274 \\
\hline 49 & 1.4562 & 3 & 30.05 & 2.56 & 0.5023 \\
\hline
\end{tabular}

Table 2 indicates the status of nodes during the simulation period. Here the random observations are taken, which indicates that the nodes have linear/nonlinear/zero motion. Moreover, each node has knowledge about its neighboring nodes. This sample table was created during simulation of MANET with 50 nodes and dynamic random movements.

Table 3. Historical Table

\begin{tabular}{|c|c|c|c|c|c|}
\hline ID ${ }^{\text {Node }}$ & $\begin{array}{l}\text { Last Updated } \\
\text { Time (ms) }\end{array}$ & $\begin{array}{l}\text { Energy } \\
\text { (joule) }\end{array}$ & $\begin{array}{c}\text { Time to Expire } \\
\text { (Within the cluster) }\end{array}$ & $\begin{array}{l}\text { Voting } \\
\text { count }\end{array}$ & $\begin{array}{l}\mathrm{CH} \\
\text { count }\end{array}$ \\
\hline${ }_{5}$ SimI0 & 0.3254 & 15.62 & 0.7892 & 5 & 0 \\
\hline $\begin{array}{ll}\text { SimI1 } \\
\end{array}$ & 0.5789 & 32.58 & 1.2356 & 9 & 4 \\
\hline${ }_{5}$ SimI2 & 0.4325 & 25.32 & 0.9256 & 7 & 1 \\
\hline $2^{\text {SimI3 }}$ & 1.2389 & 20.14 & 0.3278 & 2 & 0 \\
\hline${ }_{5}$ SimI4 & 1.7895 & 12.92 & 0.4256 & 4 & 0 \\
\hline
\end{tabular}

From the historical table, the system will assign the priority weights to the node which is to be selected as Cluster Head. For example, from the table we can find that the node (SimI13) has high energy value, high voting count, and high $\mathrm{CH}$ count. Due to this reason the system will allocate the high priority value and SimI13 will be selected as a cluster head. Moreover, the system will generate the report of number of nodes in the network, number of packets generated, number of clusters in the network, node ID, and address of the $\mathrm{CH}$ with respect to time. In figure $1 \mathrm{a}$, the system adapts three schemes which include none, CDTC and CDTC with lossy link estimation. In none scheme, the network will act as regular nodes with no topology control over network. 


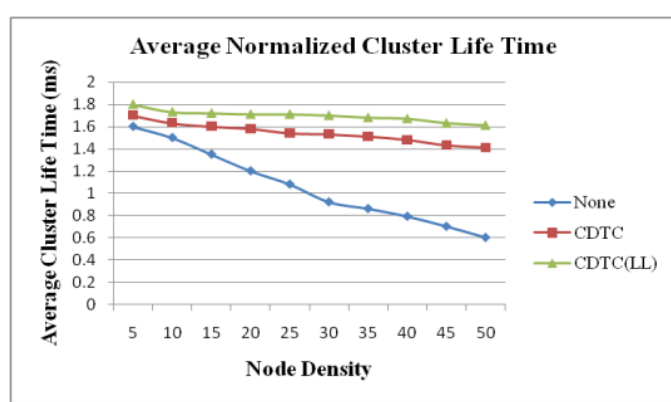

(a)

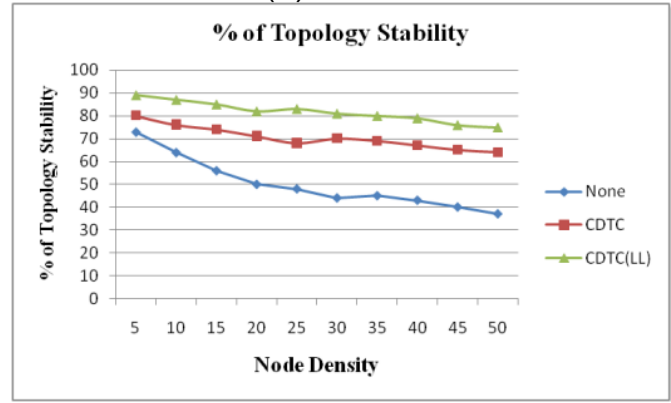

(c)

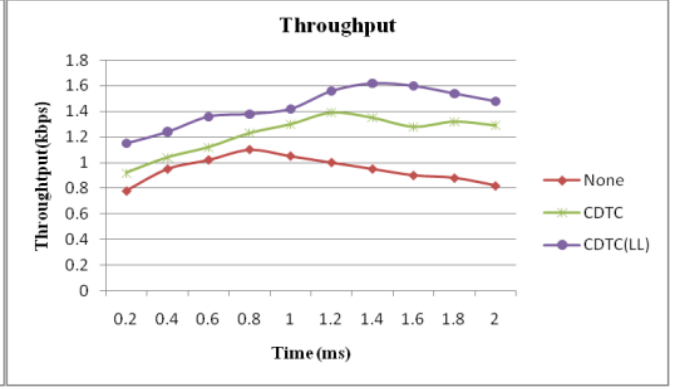

(b)

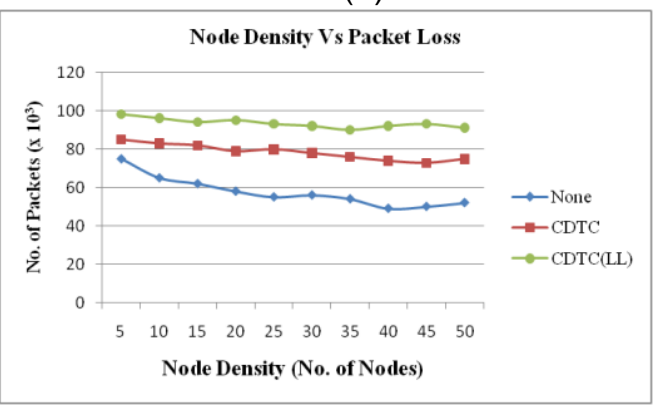

(d)

Figure 1. Performance Metrics of the MANET (A) Throughput of the Network (B) Average Normalized Cluster Life Time (C) Node Density Vs Packet Loss (D) Percentage of Topology Stability

Here no cluster has been formed and has less throughput in the network. The illustration of packet loss during the session is shown in the figure 1(b). In our scheme, the total life time of the cluster has increased compared with the existing system as shown in figure $1(\mathrm{c})$. In figure 1(d), the stability status of the network is found to be better compared with none topology. The estimation of node survivability has been achieved throughout the network and the topology stability is maintained during the session.

\section{Conclusion}

Many of the existing MANET research methods have focused on the energy management, reducing power consumption and security issues. Our proposed work provides improved topology control in dynamic MANET environment. Our network has been found to maintain at least the minimum duration of stability and the lower packet delivery loss achieved through the estimation of lossy link. The key challenges of our work were to identify the random movements of the nodes over the topography. In Awais et. al., [16,17] Energy aware and optimization are other constraints to be considered in topology management which is outlined by Sohail and Paul in $[18,19]$. The cluster heads play a major role over the network and control the cluster members thereby providing an efficient service to the network. By knowing the survivability of each node, the cluster formation and maintenance can be done efficiently. In our future work, we plan to focus on the vulnerable nodes and include security measures in the network. Our proposed framework can be made to include admission control methods for further improving the network stability and for endowing with effective topology control over the dynamic MANET environment. 


\section{Acknowledgments}

This study was supported by the Brain Korea 21 Plus project (SW Human Resource Development Program for Supporting Smart Life) funded by Ministry of Education, School of Computer Science and Engineering, Kyungpook National University, Korea (21A20131600005). This work is also supported by the IT R\&D Program of MSIP/IITP. [10041145, Self-Organized Software Platform (SoSp) for Welfare Devices].

\section{References}

[1] Y. Liu, L. Ni and Chuanping Hu, "A Generalized Probabilistic Topology Control for Wireless Sensor Networks", IEEE Journal On Selected Areas In Communications, vol. 30, no. 9, (2012), pp. 1780-1788.

[2] Q. Guan, F. R. Yu, S. Jiang and V. C. M. Leung, "Joint Topology Control and Authentication Design in Mobile Ad Hoc Networks with Cooperative Communications", IEEE Transactions on Vehicular Technology, vol. 61, no. 6, (2012), pp. 2674-2685.

[3] L. Li, J. Y. Halpern, P. Bahl, Y.-M. Wang and R. Wattenhofer, "A Cone-Based Distributed Topology-Control Algorithm for Wireless Multi-Hop Networks", IEEE/ACM Transactions on Networking, vol. 13, no. 1, (2005), pp. 147-159.

[4] C.-C. Shen, C. Srisathapornphat, R. Liu, Z. Huang, C. Jaikaeo and E. L. Lloyd, "CLTC: A ClusterBased Topology Control Framework for Ad Hoc Networks", IEEE Transactions on Mobile Computing, vol. 3, no. 1, (2004), pp. 18-32.

[5] M. N. Lima, A. L. D Santos and G. Pujolle, "A Survey of Survivability in Mobile Ad Hoc Networks", IEEE Communications Surveys \& Tutorials, vol. 11, no. 1, (2009), pp. 66-77.

[6] L. Hanzo II. and R. Tafazolli, "Admission Control Schemes for 802.11-Based Multi-Hop Mobile Ad hoc Networks: A Survey”, IEEE Communications Surveys \& Tutorials, vol. 11, no. 4, (2009), pp. 78-108.

[7] J. Kang, Y. Zhang and B. Nath, "TARA: Topology-Aware Resource Adaptation to Alleviate Congestion in Sensor Networks", IEEE Transactions on Parallel And Distributed Systems, vol. 18, no. 7, (2007), pp. 919-931.

[8] N. Li and J. C. Hou, "Localized Topology Control Algorithms for Heterogeneous Wireless Networks", IEEE/ACM Transactions on Networking, vol. 13, no. 6, (2005), pp. 1313-1324.

[9] M. Z. Zamalloa, K. Seada, B. Krishnamachari and A. Helmy, "Efficient Geographic Routing over Lossy Links in Wireless Sensor Networks", ACM Transactions on Sensor Networks, vol. 4, no. 3, (2008), pp. 111-143.

[10] Kevin H. Liu, Changdong Liu, "Performance and Testbed Study of Topology Reconfiguration in IP Over Optical Networks", IEEE Transactions on Communications, vol. 50, no. 10, (2002), pp. 16621679.

[11] Y. Liu and Q. Zhang, "Opportunity-Based Topology Control in Wireless Sensor Networks", IEEE Transactions on Parallel and Distributed Systems, vol. 21, no. 3, (2010), pp. 405-416.

[12]K. Miyao and H. Nakayama, "LTRT: An Efficient and Reliable Topology Control Algorithm for Ad-Hoc Networks", IEEE Transactions on Wireless Communications, vol. 8, no. 12, (2009), pp. $6050-6058$.

[13]N. Mohammed and H. Otrok, "Mechanism Design-Based Secure Leader Election Model for Intrusion Detection in MANET", IEEE Transactions on Dependable and Secure Computing, vol. 8, no. 1, (2011), pp. 89-103.

[14]L. Li and J. Y. Halpern, “A Minimum-Energy Path-Preserving Topology-Control Algorithm”, IEEE Transactions on Wireless Communications, vol. 3, no. 3, (2004), pp. 910-921.

[15] V. Rodoplu and T. H. Meng, "Minimum Energy Mobile Wireless Networks", IEEE Journal On Selected Areas in Communications, vol. 17, no. 8, (1999), pp. 1333-1344.

[16] A. Ahmad, A. Dainel and A. Paul, "Optimized Data Transmission Using Cooperative Devices in Clustered D2D Communication", Proceedings of the ACM Research in Adaptive and Convergent Systems, Towson, USA, (2014) October 5-8.

[17] A. Ahmad, S. Jabbar, A. Paul and S. Rho, "Mobility Aware Energy Efficient Congestion Control in Wireless Sensor Network", International Journal of Distributed Sensor Networks, vol. 2014, Article ID 530416, (2014), pp. 1-13.

[18] A. Paul, S. Rho and K. Bharanitharan, "Interactive Scheduling for Mobile Multimedia Service in M2M environment", Multimedia Tools and Application, vol. 71, no. 1, (2014), pp.235-246. 
[19]S. Jabbar, A. A. Minhas, A. Paul and S. Rho, "Multilayer Cluster Designing Algorithm for Lifetime Improvement of Wireless Sensor Networks", Journal of Supercomputing, vol. 70, no. 1, (2014), pp. 104132.

[20]B. Pazand and C. McDonald, "A Critique of Mobility Models for Wireless Network Simulation", Proceedings of the 6th IEEE/ACIS International Conference on Computer and Information Science, Los Alamitos, CA, USA, (2007) July 11-17.

[21]H. Tian and H. Shen, "Parallel and Distributed Computing: Applications and Technologies", Lecture Notes in Computer Science, vol. 3320, (2005), pp. 416-419.

[22]A. A. Aziz, Y. A. Sekercioglu, P. Fitzpatrick and M. Ivanovich, "A Survey on Distributed Topology Control Techniques for Extending the Lifetime of Battery Powered Wireless Sensor Networks", IEEE Communications Surveys \& Tutorials, vol. 15, no. 1, (2013), pp. 121-144.

[23]M. N. Lima, A. L. D. Santos and G. Pujolle, "A Survey of Survivability in Mobile Ad Hoc Networks", IEEE Communications Surveys \& Tutorials, vol. 11, no. 1, (2009), pp. 66-77.

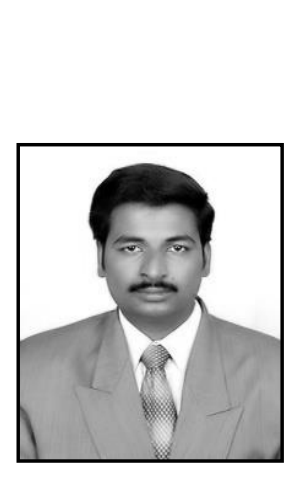

\section{Authors}

Parameswaran.T has received his B.E degree in Electronics and Communication Engineering from Velalar College of Engineering and Technology, Erode, and M.E degree in Software Engineering from College of Engineering Guindy, Anna University Chennai in 2005 and 2008 respectively. He is currently pursuing his Ph.D Anna University Chennai. He is currently working as Teaching Fellow in the Department of Computer Science and Engineering, Anna University Regional Campus, Coimbatore, Tamilnadu, India.

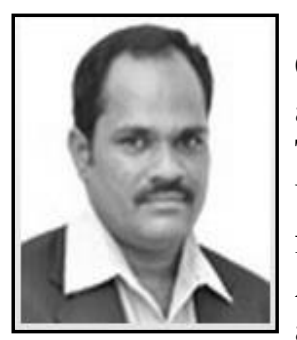

Palanisamy. C has received his B.E degree in Electronics and Communication Engineering from University of Madras, Chennai and M.E degree (Gold Medalist) in Communication Systems from Thiagarajar College of Engineering, Madurai, Madurai Kamaraj University in 1998 and 2000 respectively. He has received his Ph.D from the faculty of Information and Communication Engineering, Anna University, Chennai in 2009. He has more than 15 years of academic and research experience and currently he holds the post of Professor and Head of the Department of Information Technology, Bannari Amman Institute of Technology, Sathyamangalam, Tamilnadu, India. He has published more than 40 research papers in various journals and conferences. He has organized more than 15 workshops and holds 2 funded projects. He is a lifetime member of ISTE. He Won Best M.E Thesis Award at Thiagarajar College of Engineering, Madurai and best paper award titled, "A Neural Network Based Classification Model Using Fourier and Wavelet Features," Proceedings of the 2nd Int. Conf. on Cognition and Recognition 2008, (ICCR 2008), Organised by P. E. S. College of Engineering, Mandaya, Karnataka, India, pp. 664-670, 2008. His research interests include Data mining, image processing, and mobile networks. 


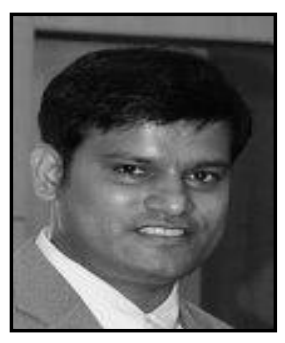

Anand Paul he is currently working in The School of Computer Science and Engineering, Kyungpook National University, South Korea as Associate Professor, He got his Ph.D. degree in the electrical engineering at National Cheng Kung University, Taiwan, R.O.C. in 2010. His research interests include Algorithm and Architecture Reconfigurable Embedded Computing. He is a delegate representing South Korea for M2M focus group and for MPEG. 2004-2010 he has been awarded Outstanding International Student Scholarship, and in 2009, 2015 he won the best paper award in national computer Symposium, in Taipei Taiwan and international conference on Softcomputing and network security, India. He has guest edited various International journals and he is also part of editorial team for Journal of Platform Technology. He serves as a reviewer for various IEEE Transactions on Circuits and Systems for Video Technology, IEEE Transaction on System, Man and Cybernatics, IEEE Sensors, ACM Transactions on Embedded Computing Systems, IET Image Processing, IET Signal Processing and IET Circuits and Systems He gave invited talk in International Symposium on Embedded Technology workshop in 2012, He is the track chair for Smart human computer interaction in ACM SAC 2016/15/14. He is also an MPEG Delegate representing South Korea

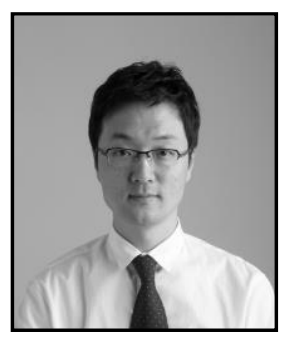

Seungmin Rho received his $\mathrm{PhD}$ degree in Computer Science from Ajou University, Korea in 2008. In 2008-2009, he was a Postdoctoral Research Fellow at the Computer Music Lab of the School of Computer Science in Carnegie Mellon University. In 20092011, he had been working as a Research Professor at School of Electrical Engineering in Korea University. In 2012, he was an assistant professor at Division of Information and Communication in Baekseok University. Dr. Rho is currently a faculty of Department of Multimedia at Sungkyul University. He is an Editor-in-Chief at Journal of Platform Technology. His current research interests include database, big data analysis, music retrieval, multimedia systems, machine learning, knowledge management as well as computational intelligence. He has published more than 200 papers in refereed journals and conference proceedings in these areas. He has been involved in more than 20 conferences and workshops as various chairs and more than 30 conferences/workshops as a program committee member. He has been appointed as an Editor-in-Chief in Journal of Platform Technology since 2013. He has edited a number of international journal special issues as a guest editor, such as Enterprise Information Systems, Multimedia Systems, Information Fusion, ACM Transactions on Embedded Computing, Journal of Real-Time Image Processing, Future Generation Computer Systems, Engineering Applications of Artificial Intelligence, New Review of Hypermedia and Multimedia, Multimedia Tools and Applications, Personal and Ubiquitous Computing, Telecommunication Systems, Ad Hoc \& Sensor Wireless Networks and etc. He has received a few awards including Who's Who in America, Who's Who in Science and Engineering, and Who's Who in the World in 2007 and 2008, respectively. 
International Journal of Control and Automation Vol.8, No.12 (2015) 